DESY-09-036

27 January 2009

\title{
Exclusive photoproduction of $\Upsilon$ mesons at HERA
}

\author{
ZEUS Collaboration
}

\begin{abstract}
The exclusive photoproduction reaction $\gamma p \rightarrow \Upsilon p$ has been studied with the ZEUS experiment in ep collisions at HERA using an integrated luminosity of $468 \mathrm{pb}^{-1}$. The measurement covers the kinematic range $60<W<220 \mathrm{GeV}$ and $Q^{2}<1 \mathrm{GeV}^{2}$, where $W$ is the photon-proton centre-of-mass energy and $Q^{2}$ is the photon virtuality. These results, which represent the analysis of the full ZEUS data sample for this channel, are compared to predictions based on perturbative QCD.
\end{abstract}




\section{The ZEUS Collaboration}

S. Chekanov, M. Derrick, S. Magill, B. Musgrave, D. Nicholass ${ }^{1}$, J. Repond, R. Yoshida Argonne National Laboratory, Argonne, Illinois 60439-4815, USA ${ }^{n}$

M.C.K. Mattingly

Andrews University, Berrien Springs, Michigan 49104-0380, USA

P. Antonioli, G. Bari, L. Bellagamba, D. Boscherini, G. Bruni, F. Cindolo, M. Corradi,

G. Iacobucci, A. Margotti, R. Nania, A. Polini

INFN Bologna, Bologna, Italy ${ }^{e}$

S. Antonelli, M. Basile, M. Bindi, L. Cifarelli, A. Contin, S. De Pasquale ${ }^{2}$, G. Sartorelli, A. Zichichi

University and INFN Bologna, Bologna, Italy ${ }^{e}$

D. Bartsch, I. Brock, H. Hartmann, E. Hilger, H.-P. Jakob, M. Jüngst, A.E. Nuncio-Quiroz,

E. Paul, U. Samson, V. Schönberg, R. Shehzadi, M. Wlasenko

Physikalisches Institut der Universität Bonn, Bonn, Germany ${ }^{b}$

N.H. Brook, G.P. Heath, J.D. Morris

H.H. Wills Physics Laboratory, University of Bristol, Bristol, United Kingdom ${ }^{m}$

M. Kaur, P. Kaur ${ }^{3}$, I. Singh ${ }^{3}$

Panjab University, Department of Physics, Chandigarh, India

M. Capua, S. Fazio, A. Mastroberardino, M. Schioppa, G. Susinno, E. Tassi

Calabria University, Physics Department and INFN, Cosenza, Italy ${ }^{e}$

J.Y. Kim

Chonnam National University, Kwangju, South Korea

Z.A. Ibrahim, F. Mohamad Idris, B. Kamaluddin, W.A.T. Wan Abdullah

Jabatan Fizik, Universiti Malaya, 50603 Kuala Lumpur, Malaysia ${ }^{r}$

Y. Ning, Z. Ren, F. Sciulli

Nevis Laboratories, Columbia University, Irvington on Hudson, New York 10027, USA ${ }^{\circ}$

J. Chwastowski, A. Eskreys, J. Figiel, A. Galas, K. Olkiewicz, B. Pawlik, P. Stopa,

L. Zawiejski

The Henryk Niewodniczanski Institute of Nuclear Physics, Polish Academy of Sciences, Cracow, Poland ${ }^{i}$

L. Adamczyk, T. Bołd, I. Grabowska-Bołd, D. Kisielewska, J. Łukasik ${ }^{4}$, M. Przybycień,

L. Suszycki

Faculty of Physics and Applied Computer Science, AGH-University of Science and Technology, Cracow, Poland ${ }^{p}$ 
A. Kotański ${ }^{5}$, W. Słomiński ${ }^{6}$

Department of Physics, Jagellonian University, Cracow, Poland

O. Behnke, J. Behr, U. Behrens, C. Blohm, K. Borras, D. Bot, R. Ciesielski, N. Coppola, S. Fang, A. Geiser, P. Göttlicher ${ }^{7}$, J. Grebenyuk, I. Gregor, T. Haas, W. Hain, A. Hüttmann, F. Januschek, B. Kahle, I.I. Katkov ${ }^{8}$, U. Klein ${ }^{9}$, U. Kötz, H. Kowalski, M. Lisovyi, E. Lobodzinska, B. Löhr, R. Mankel ${ }^{10}$, I.-A. Melzer-Pellmann, S. Miglioranzi ${ }^{11}$, A. Montanari, T. Namsoo, D. Notz, A. Parenti, P. Roloff, I. Rubinsky, U. Schneekloth, A. Spiridonov ${ }^{12}$, D. Szuba ${ }^{13}$, J. Szuba ${ }^{14}$, T. Theedt, J. Tomaszewska ${ }^{15}$, G. Wolf, K. Wrona, A.G. Yagües-Molina, C. Youngman, W. Zeuner ${ }^{10}$

Deutsches Elektronen-Synchrotron DESY, Hamburg, Germany

V. Drugakov, W. Lohmann, S. Schlenstedt

Deutsches Elektronen-Synchrotron DESY, Zeuthen, Germany

G. Barbagli, E. Gallo

INFN Florence, Florence, Italy ${ }^{e}$

P. G. Pelfer

University and INFN Florence, Florence, Italy $^{e}$

A. Bamberger, D. Dobur, F. Karstens, N.N. Vlasov ${ }^{16}$

Fakultät für Physik der Universität Freiburg i.Br., Freiburg i.Br., Germany ${ }^{b}$

P.J. Bussey, A.T. Doyle, M. Forrest, D.H. Saxon, I.O. Skillicorn

Department of Physics and Astronomy, University of Glasgow, Glasgow, United Kingdom ${ }^{m}$

I. Gialas ${ }^{17}$, K. Papageorgiu

Department of Engineering in Management and Finance, Univ. of Aegean, Greece

U. Holm, R. Klanner, E. Lohrmann, H. Perrey, P. Schleper, T. Schörner-Sadenius, J. Sztuk, H. Stadie, M. Turcato

Hamburg University, Institute of Exp. Physics, Hamburg, Germany ${ }^{b}$

C. Foudas, C. Fry, K.R. Long, A.D. Tapper

Imperial College London, High Energy Nuclear Physics Group, London, United Kingdom ${ }^{m}$

T. Matsumoto, K. Nagano, K. Tokushuku ${ }^{18}$, S. Yamada, Y. Yamazaki ${ }^{19}$

Institute of Particle and Nuclear Studies, KEK, Tsukuba, Japan ${ }^{f}$

A.N. Barakbaev, E.G. Boos, N.S. Pokrovskiy, B.O. Zhautykov

Institute of Physics and Technology of Ministry of Education and Science of Kazakhstan, Almaty, Kazakhstan 
V. Aushev ${ }^{20}$, O. Bachynska, M. Borodin, I. Kadenko, O. Kuprash, V. Libov, D. Lontkovskyi, I. Makarenko, Iu. Sorokin, A. Verbytskyi, O. Volynets, M. Zolko

Institute for Nuclear Research, National Academy of Sciences, and Kiev National University, Kiev, Ukraine

D. Son

Kyungpook National University, Center for High Energy Physics, Daegu, South Korea ${ }^{g}$

J. de Favereau, K. Piotrzkowski

Institut de Physique Nucléaire, Université Catholique de Louvain, Louvain-la-Neuve, Belgium ${ }^{q}$

F. Barreiro, C. Glasman, M. Jimenez, J. del Peso, E. Ron, J. Terrón, C. Uribe-Estrada Departamento de Física Teórica, Universidad Autónoma de Madrid, Madrid, Spain ${ }^{l}$

F. Corriveau, J. Schwartz, C. Zhou

Department of Physics, McGill University, Montréal, Québec, Canada H3A 2T8 ${ }^{a}$

T. Tsurugai

Meiji Gakuin University, Faculty of General Education, Yokohama, Japan ${ }^{f}$

A. Antonov, B.A. Dolgoshein, D. Gladkov, V. Sosnovtsev, A. Stifutkin, S. Suchkov Moscow Engineering Physics Institute, Moscow, Russia ${ }^{j}$

R.K. Dementiev, P.F. Ermolov ${ }^{\dagger}$, L.K. Gladilin, Yu.A. Golubkov, L.A. Khein, I.A. Korzhavina, V.A. Kuzmin, B.B. Levchenko ${ }^{21}$, O.Yu. Lukina, A.S. Proskuryakov, L.M. Shcheglova, D.S. Zotkin

Moscow State University, Institute of Nuclear Physics, Moscow, Russia ${ }^{k}$

I. Abt, A. Caldwell, D. Kollar, B. Reisert, W.B. Schmidke

Max-Planck-Institut für Physik, München, Germany

G. Grigorescu, A. Keramidas, E. Koffeman, P. Kooijman, A. Pellegrino, H. Tiecke, M. Vázquez ${ }^{11}$, L. Wiggers

NIKHEF and University of Amsterdam, Amsterdam, Netherlands ${ }^{h}$

N. Brümmer, B. Bylsma, L.S. Durkin, A. Lee, T.Y. Ling

Physics Department, Ohio State University, Columbus, Ohio 43210, USA ${ }^{n}$

P.D. Allfrey, M.A. Bell, A.M. Cooper-Sarkar, R.C.E. Devenish, J. Ferrando, B. Foster,

C. Gwenlan ${ }^{22}$, K. Horton ${ }^{23}$, K. Oliver, A. Robertson, R. Walczak

Department of Physics, University of Oxford, Oxford United Kingdom ${ }^{m}$

A. Bertolin, F. Dal Corso, S. Dusini, A. Longhin, L. Stanco

INFN Padova, Padova, Italy ${ }^{e}$

R. Brugnera, R. Carlin, A. Garfagnini, S. Limentani

Dipartimento di Fisica dell' Università and INFN, Padova, Italy ${ }^{e}$ 
B.Y. Oh, A. Raval, J.J. Whitmore ${ }^{24}$

Department of Physics, Pennsylvania State University, University Park, Pennsylvania $16802^{\circ}$

Y. Iga

Polytechnic University, Sagamihara, Japan ${ }^{f}$

G. D'Agostini, G. Marini, A. Nigro

Dipartimento di Fisica, Università 'La Sapienza' and INFN, Rome, Italy ${ }^{e}$

J.E. Cole ${ }^{25}$, J.C. Hart

Rutherford Appleton Laboratory, Chilton, Didcot, Oxon, United Kingdom ${ }^{m}$

H. Abramowicz ${ }^{26}$, R. Ingbir, S. Kananov, A. Levy, A. Stern

Raymond and Beverly Sackler Faculty of Exact Sciences, School of Physics, Tel Aviv

University,

Tel Aviv, Israel ${ }^{d}$

M. Kuze, J. Maeda

Department of Physics, Tokyo Institute of Technology, Tokyo, Japan ${ }^{f}$

R. Hori, S. Kagawa ${ }^{27}$, N. Okazaki, S. Shimizu, T. Tawara

Department of Physics, University of Tokyo, Tokyo, Japan ${ }^{f}$

R. Hamatsu, H. Kaji ${ }^{28}$, S. Kitamura ${ }^{29}$, O. Ota ${ }^{30}$, Y.D. Ri

Tokyo Metropolitan University, Department of Physics, Tokyo, Japan ${ }^{f}$

M. Costa, M.I. Ferrero, V. Monaco, R. Sacchi, V. Sola, A. Solano

Università di Torino and INFN, Torino, Italy ${ }^{e}$

M. Arneodo, M. Ruspa

Università del Piemonte Orientale, Novara, and INFN, Torino, Italy ${ }^{e}$

S. Fourletov ${ }^{31}$, J.F. Martin, T.P. Stewart

Department of Physics, University of Toronto, Toronto, Ontario, Canada M5S 1A7 a

S.K. Boutle ${ }^{17}$, J.M. Butterworth, T.W. Jones, J.H. Loizides, M. Wing ${ }^{32}$

Physics and Astronomy Department, University College London, London, United Kingdom ${ }^{m}$

B. Brzozowska, J. Ciborowski ${ }^{33}$, G. Grzelak, P. Kulinski, P. Łużniak ${ }^{34}$, J. Malka ${ }^{34}$, R.J. Nowak, J.M. Pawlak, W. Perlanski ${ }^{34}$, A.F. Żarnecki

Warsaw University, Institute of Experimental Physics, Warsaw, Poland

M. Adamus, P. Plucinski ${ }^{35}$

Institute for Nuclear Studies, Warsaw, Poland

Y. Eisenberg, D. Hochman, U. Karshon

Department of Particle Physics, Weizmann Institute, Rehovot, Israel ${ }^{c}$ 
E. Brownson, D.D. Reeder, A.A. Savin, W.H. Smith, H. Wolfe

Department of Physics, University of Wisconsin, Madison, Wisconsin 53706, USA ${ }^{n}$

S. Bhadra, C.D. Catterall, Y. Cui, G. Hartner, S. Menary, U. Noor, J. Standage, J. Whyte Department of Physics, York University, Ontario, Canada M3J 1P3 ${ }^{a}$ 
${ }^{1}$ also affiliated with University College London, United Kingdom

2 now at University of Salerno, Italy

3 also working at Max Planck Institute, Munich, Germany

${ }^{4}$ now at Institute of Aviation, Warsaw, Poland

${ }^{5}$ supported by the research grant No. 1 P03B 04529 (2005-2008)

6 This work was supported in part by the Marie Curie Actions Transfer of Knowledge project COCOS (contract MTKD-CT-2004-517186)

${ }^{7}$ now at DESY group FEB, Hamburg, Germany

8 also at Moscow State University, Russia

${ }^{9}$ now at University of Liverpool, UK

10 on leave of absence at CERN, Geneva, Switzerland

11 now at CERN, Geneva, Switzerland

12 also at Institut of Theoretical and Experimental Physics, Moscow, Russia

13 also at INP, Cracow, Poland

14 also at FPACS, AGH-UST, Cracow, Poland

15 partially supported by Warsaw University, Poland

16 partly supported by Moscow State University, Russia

17 also affiliated with DESY, Germany

18 also at University of Tokyo, Japan

19 now at Kobe University, Japan

20 supported by DESY, Germany

21 partly supported by Russian Foundation for Basic Research grant No. 05-02-39028NSFC-a

22 STFC Advanced Fellow

23 nee Korcsak-Gorzo

${ }^{24}$ This material was based on work supported by the National Science Foundation, while working at the Foundation.

25 now at University of Kansas, Lawrence, USA

26 also at Max Planck Institute, Munich, Germany, Alexander von Humboldt Research Award

27 now at KEK, Tsukuba, Japan

28 now at Nagoya University, Japan

29 member of Department of Radiological Science, Tokyo Metropolitan University, Japan

30 now at SunMelx Co. Ltd., Tokyo, Japan

31 now at University of Bonn, Germany

32 also at Hamburg University, Inst. of Exp. Physics, Alexander von Humboldt Research Award and partially supported by DESY, Hamburg, Germany

33 also at Łódź University, Poland

${ }^{34}$ member of Łódź University, Poland

35 now at Lund University, Lund, Sweden

$\dagger$ deceased 
a supported by the Natural Sciences and Engineering Research Council of Canada (NSERC)

$b$ supported by the German Federal Ministry for Education and Research (BMBF), under contract Nos. 05 HZ6PDA, 05 HZ6GUA, 05 HZ6VFA and 05 HZ4KHA

c supported in part by the MINERVA Gesellschaft für Forschung GmbH, the Israel Science Foundation (grant No. 293/02-11.2) and the US-Israel Binational Science Foundation

$d \quad$ supported by the Israel Science Foundation

$e$ supported by the Italian National Institute for Nuclear Physics (INFN)

$f$ supported by the Japanese Ministry of Education, Culture, Sports, Science and Technology (MEXT) and its grants for Scientific Research

$g$ supported by the Korean Ministry of Education and Korea Science and Engineering Foundation

$h \quad$ supported by the Netherlands Foundation for Research on Matter (FOM)

$i$ supported by the Polish State Committee for Scientific Research, project No. DESY/256/2006 - 154/DES/2006/03

$j$ partially supported by the German Federal Ministry for Education and Research $(\mathrm{BMBF})$

$k$ supported by RF Presidential grant N 1456.2008.2 for the leading scientific schools and by the Russian Ministry of Education and Science through its grant for Scientific Research on High Energy Physics

$l$ supported by the Spanish Ministry of Education and Science through funds provided by CICYT

$m$ supported by the Science and Technology Facilities Council, UK

$n$ supported by the US Department of Energy

$o$ supported by the US National Science Foundation. Any opinion, findings and conclusions or recommendations expressed in this material are those of the authors and do not necessarily reflect the views of the National Science Foundation.

$p \quad$ supported by the Polish Ministry of Science and Higher Education as a scientific project (2006-2008)

$q$ supported by FNRS and its associated funds (IISN and FRIA) and by an Inter-University Attraction Poles Programme subsidised by the Belgian Federal Science Policy Office

$r$ supported by an FRGS grant from the Malaysian government 


\section{Introduction}

Exclusive photoproduction of heavy vector mesons, $J / \psi$ and $\Upsilon$, has previously been studied at HERA [1-4]. The process $\gamma p \rightarrow V p$, with $V=J / \psi, \Upsilon$ can be described by perturbative QCD (pQCD), since the relatively high masses of the charm and bottom quarks provide sufficiently hard scales. The process is assumed to exhibit threefold factorisation [5-8]: the photon fluctuates into a $q \bar{q}$ pair; the pair interacts with the proton; and finally the heavy meson is formed in the final state. At leading order (LO), the interaction of the $q \bar{q}$ pair with the proton proceeds via the exchange of two gluons in a colour-singlet state. Thus the cross section is proportional to the square of the gluon density in the proton. The rise of the gluon density with decreasing fractional momentum, $x$, leads to the prediction of a cross section rapidly rising as a function of the photonproton centre-of-mass energy, $W$, where the relevant $x$ region accessible in heavy-quark production at HERA is $10^{-4}<x<10^{-2}$. The rise of the cross section, $\sigma$, with $W$ can be expressed as $\sigma \propto W^{\delta}$, where a value of $\delta \approx 0.7-0.8$ has been measured for the $J / \psi[1,4]$. A value of 1.7 is predicted for $\Upsilon(1 \mathrm{~S})$ production in LO [5].

Prior to this analysis, ZEUS and H1 measured the $\Upsilon$ photoproduction cross section for one value of $W$. The increased statistics of the data used in this study allows the investigation of the dependence of the production cross section on the energy $W$. The data cover the kinematic range $60<W<220 \mathrm{GeV}$ and the results are obtained using the $\mu^{+} \mu^{-}$decay channel. In this measurement the three upsilon states $\Upsilon(1 S), \Upsilon(2 S)$ and $\Upsilon$ (3S) (denoted $\Upsilon_{i}, i=1,2,3$, respectively) are not resolved. Hence the sum of the cross sections multiplied by the corresponding decay branching ratios to muons, $\sum_{i} \sigma^{\gamma p \rightarrow \Upsilon_{i} p} \cdot \mathcal{B}_{i}$, was measured as a function of $W$ for two intervals: $60<W<130 \mathrm{GeV}$ and $130<W<220 \mathrm{GeV}$. The sample under study represents more than a ten-fold increase in integrated luminosity compared to the previous ZEUS publication [2].

\section{Experimental set-up}

In 1998-2007 (1996-1997), HERA provided electron ${ }^{1}$ beams of energy $E_{e}=27.5 \mathrm{GeV}$ and proton beams of energy $E_{p}=920(820) \mathrm{GeV}$, resulting in centre-of-mass energies of $\sqrt{s}=318(300) \mathrm{GeV}$, corresponding to integrated luminosities of 430 (38) $\mathrm{pb}^{-1}$.

A detailed description of the ZEUS detector can be found elsewhere [9]. A brief outline of the components that are most relevant for this analysis is given below.

In the kinematic range of the analysis, charged particles were tracked in the central

\footnotetext{
${ }^{1}$ Electrons and positrons are both referred to as electrons in this paper.
} 
tracking detector (CTD) [10] and, for the data taken after 2001, also in the microvertex detector (MVD) [11]. These components operated in a magnetic field of $1.43 \mathrm{~T}$ provided by a thin superconducting solenoid. The CTD consisted of 72 cylindrical drift chamber layers, organised in nine superlayers covering the polar-angle ${ }^{2}$ region $15^{\circ}<\theta<164^{\circ}$. The MVD provided polar angle coverage from $7^{\circ}$ to $150^{\circ}$.

The high-resolution uranium-scintillator calorimeter (CAL) [12] consisted of three parts: the forward (FCAL), the barrel (BCAL) and the rear (RCAL) calorimeters. Each part was subdivided transversely into towers and longitudinally into one electromagnetic section (EMC) and either one (in RCAL) or two (in BCAL and FCAL) hadronic sections (HAC). The smallest subdivision of the calorimeter is called a cell. The CAL energy resolutions, as measured under test-beam conditions, are $\sigma(E) / E=0.18 / \sqrt{E}$ for electrons and $\sigma(E) / E=0.35 / \sqrt{E}$ for hadrons $(E$ in $\mathrm{GeV})$.

The muon system consisted of barrel, rear (B/RMUON) [13] and forward (FMUON) [9] tracking detectors. The B/RMUON consisted of limited-streamer (LS) tube chambers placed behind the BCAL (RCAL), both inside and outside the magnetised iron yoke surrounding the CAL. The barrel and rear muon chambers covered polar angles from $34^{\circ}$ to $135^{\circ}$ and from $135^{\circ}$ to $171^{\circ}$, respectively. The FMUON consisted of six planes of LS tubes and four planes of drift chambers covering the angular region from $5^{\circ}$ to $32^{\circ}$. The muon system exploited the magnetic field of the iron yoke and, in the forward direction, of two iron toroids magnetised to $1.6 \mathrm{~T}$ to provide an independent measurement of the muon momentum.

The iron yoke surrounding the CAL was instrumented with proportional drift chambers to form the Backing Calorimeter (BAC) $[14,15]$. The BAC provided position information for muon reconstruction with a resolution of few $\mathrm{mm}$ for two of its coordinates and approximately $15 \mathrm{~cm}$ for the third, covering the full angular range.

The luminosity was measured using the Bethe-Heitler reaction $e p \rightarrow e \gamma p$ with the luminosity detector which consisted of independent lead-scintillator calorimeter [16] and magnetic spectrometer [17] systems.

\footnotetext{
${ }^{2}$ The ZEUS coordinate system is a right-handed Cartesian system, with the $Z$ axis pointing in the proton beam direction, referred to as the "forward direction", and the $X$ axis pointing left towards the centre of HERA. The coordinate origin is at the nominal interaction point. The polar angle, $\theta$, is measured with respect to the proton beam direction.
} 


\section{Kinematics}

The four-momenta of the incoming electron and proton, and the scattered electron and proton are denoted by $k, p, k^{\prime}$ and $p^{\prime}$, respectively. The exclusive reaction

$$
e p \rightarrow e \Upsilon p \rightarrow e \mu^{+} \mu^{-} p
$$

at a given $s=(k+p)^{2}$ for electrons and protons, is described by the following variables:

- $Q^{2}=-q^{2}=-\left(k-k^{\prime}\right)^{2}$, the negative four-momentum squared of the exchanged photon;

- $y=(q \cdot p) /(k \cdot p)$, the fraction of the electron energy transferred to the hadronic final state in the rest frame of the initial-state proton;

- $W^{2}=(q+p)^{2}=-Q^{2}+2 y(k \cdot p)+M_{p}^{2}$, the centre-of-mass energy squared of the photon-proton system, where $M_{p}$ is the proton mass;

- $M_{\mu^{+} \mu^{-}}$, the invariant mass of the $\mu^{+} \mu^{-}$pair.

Selected events (see Section 4) are restricted to $Q^{2}$ values from the kinematic minimum, $Q_{\min }^{2}=M_{e}^{2} y^{2} /(1-y) \approx 10^{-9} \mathrm{GeV}^{2}$, for $y=0.2$, where $M_{e}$ is the electron mass, to a value at which the scattered electron starts to be observed in the CAL, $Q_{\max }^{2} \approx 1 \mathrm{GeV}^{2}$. The median $Q^{2}$ value is $10^{-3} \mathrm{GeV}^{2}$ and the photon-proton centre-of-mass energy can be expressed as

$$
W^{2} \approx 4 E_{p} E_{e} y \approx 2 E_{p}\left(E-p_{Z}\right),
$$

where $\left(E-p_{Z}\right)$ is the difference between the energy and the longitudinal momentum of the $\mu^{+} \mu^{-}$pair.

\section{Event selection}

Exclusive $\mu^{+} \mu^{-}$events in photoproduction were selected using dedicated triggers and offline selection cuts. At the trigger level, at least one CTD track matched with a F/B/RMUON deposit was required. The offline selection was [18]:

- CAL timing and vertex position consistent with a nominal ep interaction;

- two oppositely charged tracks matched to the vertex and no other track in the central tracking system;

- at least one track identified as a muon according to a procedure which uses information from B/RMUON, FMUON or BAC, whenever available in a given event [19]; if not explicitly identified as a muon, the second track had to be consistent with a minimumionising particle; 
- tracks with hits in at least 5 CTD superlayers, to ensure a good momentum resolution;

- $\left|\eta_{1}-\eta_{2}\right| \leq 1.5$, where $\eta_{i}$ is the pseudorapidity ${ }^{3}$ of a given track, to reduce the influence of the purely electromagnetic Bethe-Heitler background;

- transverse momentum of a track $p_{T}>1.5 \mathrm{GeV}$;

- a cut $\left|\pi-\theta_{1}-\theta_{2}\right| \geq 0.1$, where $\theta_{i}$ is the polar angle of a given track, to further reject cosmic-ray events;

- invariant mass $M_{\mu^{+} \mu^{-}}$in the range between 5 and $15 \mathrm{GeV}$;

- the energy of each CAL cluster not associated to any of the final-state muons to be less than $0.5 \mathrm{GeV}$. This threshold was set to be above the noise level of the CAL. It implicitly selected exclusive events with an effective cut $Q^{2}<1 \mathrm{GeV}^{2}$;

- to suppress the contamination from proton-dissociative events, $e p \rightarrow e \Upsilon Y$, the sum of the energy in the FCAL surrounding the beam hole had to be smaller than $1 \mathrm{GeV}$ [18]. This corresponds to an effective cut on the mass $M_{Y}$ of the dissociated system, $M_{Y} \lesssim 4 \mathrm{GeV}$.

The events were selected in the kinematic range $60<W<220 \mathrm{GeV}$.

\section{Monte Carlo simulation}

The detector and trigger acceptance and the effects due to imperfect detector response were determined using samples of Monte Carlo (MC) events. Vector-meson production was simulated using the DIFFVM 2.0 generator [20] which is based on Regge phenomenology and the Vector Dominance Model in which the photon fluctuates into a virtual vector meson which interacts diffractively with the proton via Pomeron exchange. For exclusive vector-meson production, $s$-channel helicity conservation (SCHC) was assumed. An exponential dependence $\sim e^{b t}$ was assumed for the differential cross section in the fourmomentum-transfer squared distribution at the proton vertex, $t$, with a slope parameter $b=4.5 \mathrm{GeV}^{-2}$, consistent with the value obtained for exclusive $J / \psi$ electroproduction $[1,4]$. The $W$ dependence of the $\gamma p \rightarrow \Upsilon p$ cross section was parameterised as $\propto W^{\delta}$, with $^{4} \delta=1.2$. Radiative corrections are of the order of $1 \%$ [21] and are not included in the simulation. The DIFFVM generator supplemented by the JETSET 7.3 package [22] was used to estimate the value of $M_{Y}$ below which background events from proton dissociation, $\gamma p \rightarrow \Upsilon Y$, do not leave deposits in FCAL.

\footnotetext{
${ }^{3}$ Pseudorapidity is defined as $\eta=-\ln \left(\tan \frac{\theta}{2}\right)$.

4 This value was obtained from an iterative process of the analysis.
} 
The background, consisting of Bethe-Heitler dimuon events, a purely electromagnetic process, was simulated using the GRAPE v1.1k [23] MC program. All MC events were put through the simulation of the ZEUS detector based on the GEANT [24] program versions 3.13 (1996-2000) and 3.21 (2003-2007) and were analysed with the same reconstruction and offline procedures as the data. In addition, corrections $[18,19]$ of the muon detector efficiencies determined from a data set consisting of isolated $J / \psi$ and Bethe-Heitler events were applied.

\section{Signal extraction}

The distributions of the $\mu^{+} \mu^{-}$invariant mass for the selected events are presented in Fig. 1 for the two $W$ intervals, $60<W<130 \mathrm{GeV}$ and $130<W<220 \mathrm{GeV}$, and for the entire sample. A clear signal is seen around $9.5 \mathrm{GeV}$.

The mass resolution in the $\Upsilon$ resonance region is approximately $0.2 \mathrm{GeV}$ and does not allow the $\Upsilon_{i}, i=1,2,3$, states to be resolved. The number of signal events in each $W$ interval was determined using the following method. The MC of the Bethe-Heitler process was normalised to the data in a mass window not containing resonances ( 5 to $9 \mathrm{GeV}, 10.7$ to $15 \mathrm{GeV}$ ). The spectrum outside the resonance regions was reproduced and the distribution was used to obtain the signal by subtracting the background under the resonances. It was assumed that the above three states are produced at HERA in the proportions 0.73:0.19:0.08, as measured in $p \bar{p}$ collisions at the Tevatron [25]. The extracted number of signal events in the signal region $9-10.7 \mathrm{GeV}$ are tabulated in Table 1 for the different $W$ ranges. The DIFFVM generator was used to obtain the combined shape of the three $\Upsilon_{i}$ states (see Fig. 1). The DIFFVM MC was normalised to the observed number of signal events. Using the above-mentioned ratios, the number of $\Upsilon(1 S)$ candidates was calculated and is also presented in Table 1.

The procedure to determine the fraction of proton-dissociative events in the final sample, $f_{\text {pdiss }}$, has been described in detail elsewhere [1]. Due to the small rate of protondissociative $\Upsilon$ production, it was not possible to use the analysed data themselves to evaluate this fraction even on the full data sample. However, this contribution is expected to be similar in all diffractive vector meson production processes [26]. Therefore, diffractive $J / \psi$ meson production [1] was used to evaluate this correction. The value assumed in this paper was $f_{\text {pdiss }}=0.25$.

The effective photon flux calculation [27] takes into account the transverse and longitudinal flux factors [20]. Thus, the effective flux calculation depends on the assumed parameterisation $W^{\delta}$ of $\sigma(\gamma p)$. The uncertainty on the $W$ dependence is taken into account as a systematic uncertainty. 


\section{Systematic uncertainties}

The following sources of systematic uncertainty were considered [18] (numbers for the full $W$ range are given):

- signal extraction method: the background was normalised separately to the left and to the right sides of the mass spectrum outside of the signal region: ${ }_{-9}^{+1} \%$;

- the uncertainty of the CTD tracking and muon-chamber performance in the trigger, and the subsequent muon reconstruction in the offline analysis: $\pm 9 \%$;

- $f_{\text {pdiss }}$ was varied between 0.2 and 0.3 to account for uncertainties in the experimental conditions and for the fact that it was evaluated from $J / \psi$ production [1], resulting in an uncertainty of $\pm 7 \%$;

- the uncertainty related to a variation of the treatment of the calorimeter noise: $-4 \%$;

- the variation of $\delta=1.2 \pm 0.5$ results in an uncertainty of $+3.2,-2.2 \%$ on the $e p$ cross section and $-3.9 \%,+1.6 \%$ on the $\gamma p$ cross section;

- variation of the slope parameter $b=4.5 \pm 0.5 \mathrm{GeV}^{-2}$ gives a negligible effect on the acceptance.

In addition, the following uncertainties related to the $\Upsilon(1 \mathrm{~S})$ extraction were also considered:

- the $\Upsilon(1 S)$ fraction in the signal was varied within the total uncertainties quoted by the CDF collaboration [25]: $+5 \%,-6 \%$;

- the $\Upsilon(1 \mathrm{~S})$ decay branching ratio: $\pm 2.4 \%$ [28].

The total systematic uncertainty was determined by adding the individual contributions in quadrature. The values for the different $W$ ranges are given in Table 1.

The uncertainty of the luminosity determination is $2.6 \%$ and is not included in the result.

\section{Results}

All ep cross sections are given at $\sqrt{s}=318 \mathrm{GeV}$.

For each $W$ bin, the sum of $e p$ cross sections multiplied by the corresponding decay branching ratios to muons was evaluated according to the formula

$$
\sum_{i} \sigma^{\gamma p \rightarrow \Upsilon_{i} p} \cdot \mathcal{B}_{i}=\frac{N_{\mathrm{sig}}\left(1-f_{\text {pdiss }}\right)}{\mathcal{A L}},
$$


where $N_{\text {sig }}$ is the number of signal events in the signal mass region, $\mathcal{A}$ is the overall acceptance, $\mathcal{B}_{i}$ is the decay branching ratio into $\mu^{+} \mu^{-}$, and $\mathcal{L}$ is the corresponding integrated luminosity.

The $e p$ cross section for $\Upsilon(1 \mathrm{~S})$ production was calculated according to

$$
\sigma^{e p \rightarrow \Upsilon(1 S) p}=\frac{f_{\Upsilon(1 S)}}{\mathcal{B}_{1}} \sum_{i} \sigma^{\gamma p \rightarrow \Upsilon_{i} p} \cdot \mathcal{B}_{i},
$$

where $f_{\Upsilon(1 S)}=0.73_{-0.06}^{+0.05}[25]$ and $\mathcal{B}_{1}=2.48 \pm 0.06 \%[28]$.

The $\gamma p$ cross section for exclusive $\Upsilon(1 \mathrm{~S})$ photoproduction was obtained through the relation

$$
\sigma^{\gamma p \rightarrow \Upsilon(1 S) p}=\frac{1}{\Phi} \sigma^{e p \rightarrow \Upsilon(1 S) p},
$$

where $\Phi$ is the effective photon flux [18]. The following results were obtained for $Q^{2}<1$ $\mathrm{GeV}^{2}$ :

$$
\begin{aligned}
\sigma^{\gamma p \rightarrow \Upsilon(1 S) p} & =160 \pm 51_{-21}^{+48} \mathrm{pb}, \quad 60<W<130 \mathrm{GeV} \\
\sigma^{\gamma p \rightarrow \Upsilon(1 S) p} & =321 \pm 88_{-114}^{+46} \mathrm{pb}, \quad 130<W<220 \mathrm{GeV} \\
\sigma^{\gamma p \rightarrow \Upsilon(1 S) p} & =235 \pm 47_{-40}^{+30} \mathrm{pb}, \quad 60<W<220 \mathrm{GeV}
\end{aligned}
$$

The number of events, the acceptance, the flux and the cross sections in the different $W$ intervals are given in Table 1.

Figure 2 shows the extracted cross section for the two independent $W$ ranges, $60<W<$ $130 \mathrm{GeV}$ and $130<W<220 \mathrm{GeV}$. Also shown are a previous ZEUS result [2] based on a partially overlapping data set and the $\mathrm{H} 1$ result [3]. The measured two cross section values were used to calculate $\delta$, resulting in $\delta=1.2 \pm 0.8$, a value consistent with the theoretical expectation [5].

The data are compared to several theoretical calculations. Frankfurt, McDermott and Strikman (FMS) [5] based their calculation on a two-gluon exchange between the interacting $q \bar{q}$ dipoles and the proton, using CTEQ4L parton density functions (PDFs) [29]. Ivanov, Krasnikov and Szymanowski (IKS) [7] use a next-to-leading-order (NLO) calculation in which the prediction for the $W$ dependence of the cross section depends on the scale adopted for the hard scattering (presented here for $\mu=1.3$ and $7 \mathrm{GeV}$ ). Martin, Nockles, Ryskin and Teubner (MNRT) [6] have a NLO calculation using gluon densities extracted from HERA data on exclusive $J / \psi$ electroproduction. Rybarska, Schäfer and Szczurek (RSS) [8] use a $k_{T}$-factorisation approach trying a Gaussian-like and a Coulomblike light-cone wave-function for the vector meson. All the calculations are consistent with 
the data. In the IKS case, a calculation using an intermediate scale is preferred. In the RSS case, the data seem to favour a Gaussian-like wave function.

\section{Summary}

The exclusive photoproduction of $\Upsilon(1 \mathrm{~S})$ meson has been studied at HERA with the ZEUS detector in the kinematic range $60<W<220 \mathrm{GeV}, Q^{2}<1 \mathrm{GeV}^{2}$ and $\sqrt{s}=$ $318 \mathrm{GeV}$ using the muon decay channel. The dependence of the cross section on $W$ has been extracted and is in agreement with predictions of several calculations based on perturbative QCD.

\section{Acknowledgments}

We appreciate the contributions to the construction and maintenance of the ZEUS detector of many people who are not listed as authors. The HERA machine group and the DESY computing staff are especially acknowledged for their success in providing excellent operation of the collider and the data-analysis environment. We thank the DESY directorate for their strong support and encouragement. 


\section{References}

[1] ZEUS Coll., S. Chekanov et al., Eur. Phys. J. C 24, 345 (2002).

[2] ZEUS Coll., J. Breitweg et al., Phys. Lett. B 437, 432 (1998).

[3] H1 Coll., C. Adloff et al., Phys. Lett. B 483, 23 (2000).

[4] H1 Coll., A. Aktas et al., Eur. Phys. J. C 46, 585 (2006).

[5] L.L. Frankfurt, M.F. McDermott and M. Strikman,, JHEP 02, 002 (1999).

[6] A.D. Martin et al., Phys. Lett. B 662, 252 (2008).

[7] D. Yu. Ivanov, G. Krasnikov and L. Szymanowski, Nucl. Phys. Proc. Suppl. 146, 134 (2005).

[8] A. Rybarska, W. Schäfer and A. Szczurek, Phys. Lett. B 668, 126 (2008).

[9] ZEUS Coll., U. Holm (ed.), The ZEUS Detector. Status Report (unpublished), DESY (1993), available on http://www-zeus.desy.de/bluebook/bluebook.html.

[10] N. Harnew et al., Nucl. Inst. Meth. A 279, 290 (1989);

B. Foster et al., Nucl. Phys. Proc. Suppl. B 32, 181 (1993);

B. Foster et al., Nucl. Inst. Meth. A 338, 254 (1994).

[11] A. Polini et al., Nucl. Inst. Meth. A581, 31 (2007).

[12] M. Derrick et al., Nucl. Inst. Meth. A 309, 77 (1991);

A. Andresen et al., Nucl. Inst. Meth. A 309, 101 (1991);

A. Caldwell et al., Nucl. Inst. Meth. A 321, 356 (1992);

A. Bernstein et al., Nucl. Inst. Meth. A 336, 23 (1993).

[13] G. Abbiendi et al., Nucl. Instr. and Meth. A 333, 342 (1993).

[14] T. Jeżyński et al., Proceedings of SPIE - The International Society for Optical Engineering, Vol. 5484, pp. 180-184. (2004).

[15] P. Pluciński, Setup and optimisation of the Muon Trigger System for the ZEUS Backing Calorimeter. Ph.D. Thesis, The Andrzej Soltan Institute for Nuclear Studies, Warsaw (Poland), 2007, available on http://www.u.lodz.pl/polish/phd_pawel_plucinski.pdf.

[16] J. Andruszków et al., Acta Phys. Pol. B 32, 2025 (2001).

[17] M. Helbich et al., Nucl. Inst. Meth. A 565, 572 (2006).

[18] I. Rubinsky, Upsilon meson production at HERA. Ph.D. Thesis, Hamburg University, Hamburg (Germany), Report DESY-THESIS-2009-XXX, DESY, 2009. 
[19] I. Bloch, Measurement of beauty production from dimuon events at HERA/ZEUS. Ph.D. Thesis, Hamburg University, Hamburg (Germany), Report DESY-THESIS-2005-034, DESY, 2005.

[20] B. List and A. Mastroberardino, Proc. Workshop on Monte Carlo Generators for HERA Physics, p. 396. DESY, Hamburg, Germany (1999). Also in preprint DESY-PROC-1999-02, available on http://www.desy.de/ ${ }^{\sim}$ heramc/.

[21] A. Spiridonov (2005).

[22] T. Sjöstrand, Pythia 5.7 and Jetset 7.4 Physics and Manual, 1993. CERN-TH $7112 / 93$.

[23] T. Abe, Comp. Phys. Comm. 136, 126 (2001).

[24] R. Brun et al., Geant3, Technical Report CERN-DD/EE/84-1, CERN, 1987.

[25] CDF Coll., D. Acosta et al., Phys. Rev. Lett. 88, 161802 (2002).

[26] ZEUS Coll., J. Breitweg et al., Eur. Phys. J. C 14, 213 (2000).

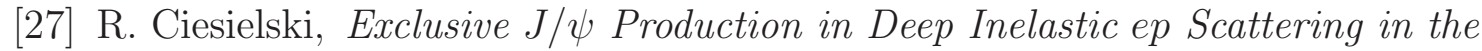
ZEUS Experiment at HERA. Ph.D. Thesis, Faculty of Physics of the Warsaw University, Institute of Experimental Physics, 2004. For the effective photon flux see formula 9.3.

[28] Particle Data Group, C. Amsler et al., Phys. Lett. B 667, 1 (2008).

[29] L.H. Lai et al., Phys. Rev. D 55, 1280 (1997). 


\begin{tabular}{||l||c|c|c||}
\hline \hline$W$ range $(\mathrm{GeV})$ & $60-130$ & $130-220$ & $60-220$ \\
\hline \hline$N_{\text {tot }}$ & $159 \pm 13$ & $135 \pm 12$ & $294 \pm 17$ \\
\hline$N_{\text {sig }}$ & $41 \pm 13$ & $44 \pm 12$ & $85 \pm 17$ \\
\hline$N_{\Upsilon(1 \mathrm{~S})}$ & $30 \pm 9$ & $32 \pm 9$ & $62 \pm 12$ \\
\hline $\mathcal{A}$ & 0.216 & 0.230 & 0.226 \\
\hline$\sum_{i} \sigma^{e p \rightarrow \Upsilon_{i} p} \cdot \mathcal{B}_{i}(p b)$ & $0.30 \pm 0.10_{-0.04}^{+0.09}$ & $0.31 \pm 0.08_{-0.11}^{+0.04}$ & $0.60 \pm 0.12_{-0.09}^{+0.07}$ \\
\hline$\sigma^{e p \rightarrow \Upsilon(1 S) p}(p b)$ & $8.9 \pm 2.8_{-1.2}^{+2.7}$ & $9.0 \pm 2.4_{-3.2}^{+1.3}$ & $17.6 \pm 3.5_{-2.9}^{+2.3}$ \\
\hline$\Phi$ & 0.055 & 0.028 & 0.074 \\
\hline$W_{0}(\mathrm{GeV})$ & 100 & 180 & 140 \\
\hline$\sum_{i} \sigma^{\gamma p \rightarrow \Upsilon_{i} p} \cdot \mathcal{B}_{i}(p b)$ & $5.5 \pm 1.8_{-0.6}^{+1.6}$ & $10.9 \pm 3.0_{-3.8}^{+1.5}$ & $8.1 \pm 1.6_{-1.3}^{+0.9}$ \\
\hline$\sigma^{\gamma p \rightarrow \Upsilon(1 S) p}(p b)$ & $160 \pm 51_{-21}^{+48}$ & $321 \pm 88_{-114}^{+46}$ & $235 \pm 47_{-40}^{+30}$ \\
\hline \hline
\end{tabular}

Table 1: The $\Upsilon$ production cross section for $Q^{2}<1$ GeV $V^{2}$. The first uncertainty is statistical, the second systematic. $N_{\text {tot }}$ is the total number of events in the signal mass region, $N_{\mathrm{sig}}$ is the number of signal events in the signal mass region, $N_{\Upsilon(1 \mathrm{~S})}$ is the extracted number of $\Upsilon(1 S)$ signal events, $\mathcal{A}$ is the acceptance, $\mathcal{B}_{i}$ is the decay branching ratio to muons of $\Upsilon_{i}, \Phi$ is the effective photon flux used to compute the $\gamma p$ cross section from the ep cross section at $W_{0}$ and a median $Q_{0}^{2}=10^{-3} \mathrm{GeV}^{2}$. 


\section{ZEUS}

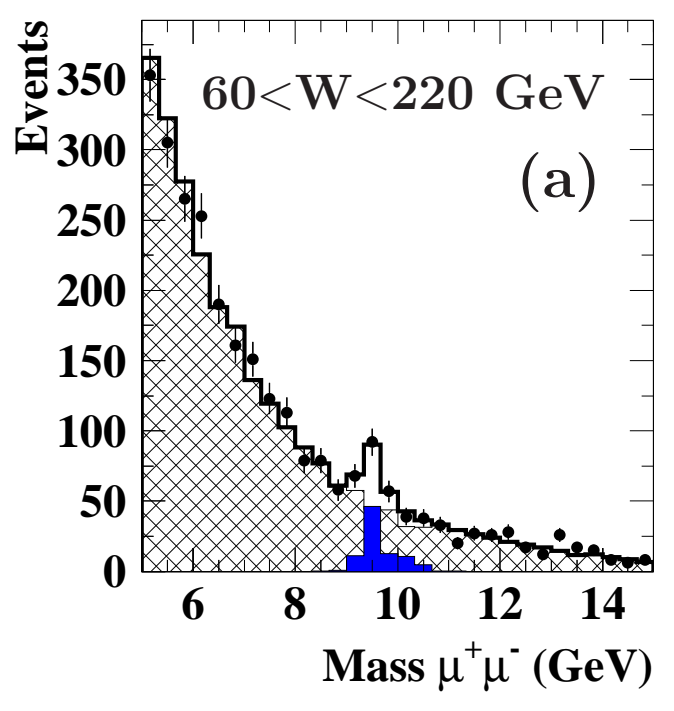

Z ZEUS 96/07 (468 pb $\left.^{-1}\right)$

GRAPE $\gamma \rightarrow \mu^{+} \mu^{-}(\mathbf{B H})$

DIFFVM $\Upsilon \rightarrow \mu^{+} \mu^{-}$

$-\mathbf{B H}+\Upsilon$
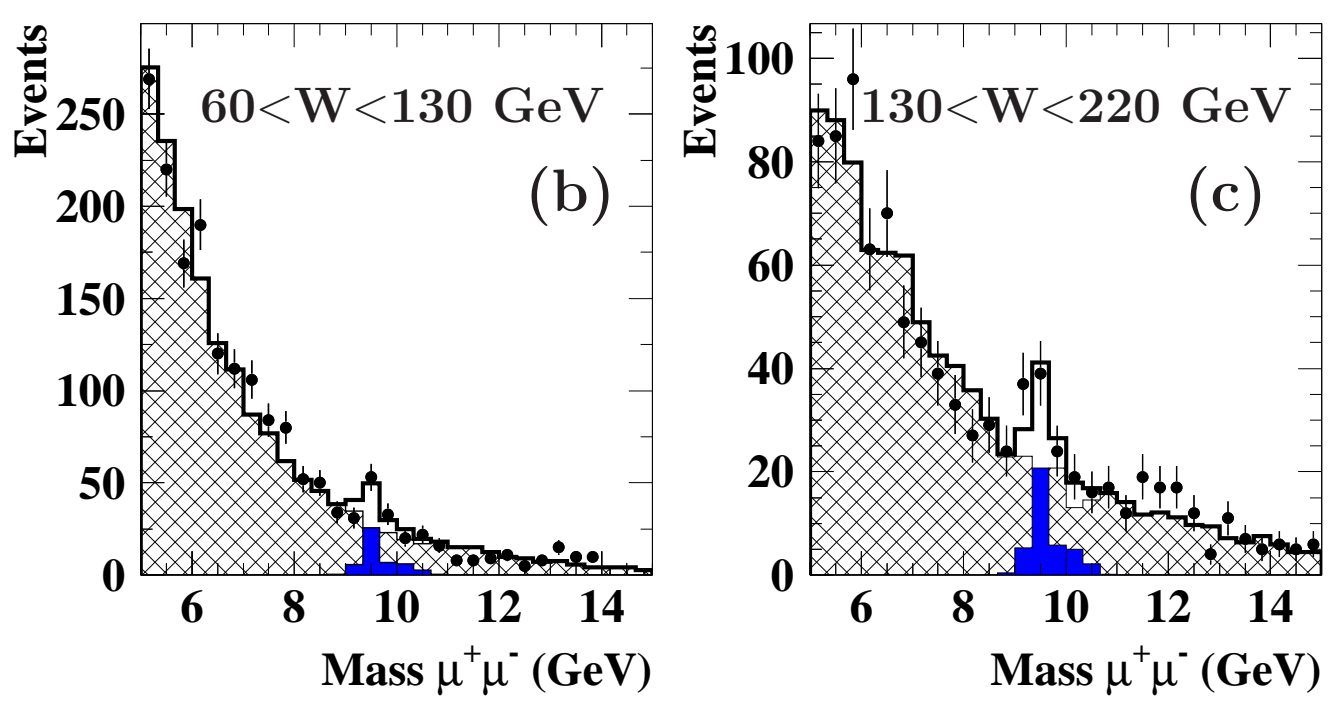

Figure 1: Invariant mass distributions of the $\mu^{+} \mu^{-}$pairs in different $W$ regions: (a) $60<W<220 \mathrm{GeV}$, (b) $60<W<130 \mathrm{GeV}$ and (c) $130<W<220 \mathrm{GeV}$. The full dots are the ZEUS data. The hatched and full histograms represent, respectively: the GRAPE distribution of the Bethe-Heitler (BH) background and the sum of DIFFVM distributions of the $\Upsilon(1 \mathrm{~S}), \Upsilon(2 \mathrm{~S})$ and $\Upsilon(3 \mathrm{~S})$ signals. The solid line is the sum of the two contributions (see text). 


\section{ZEUS}

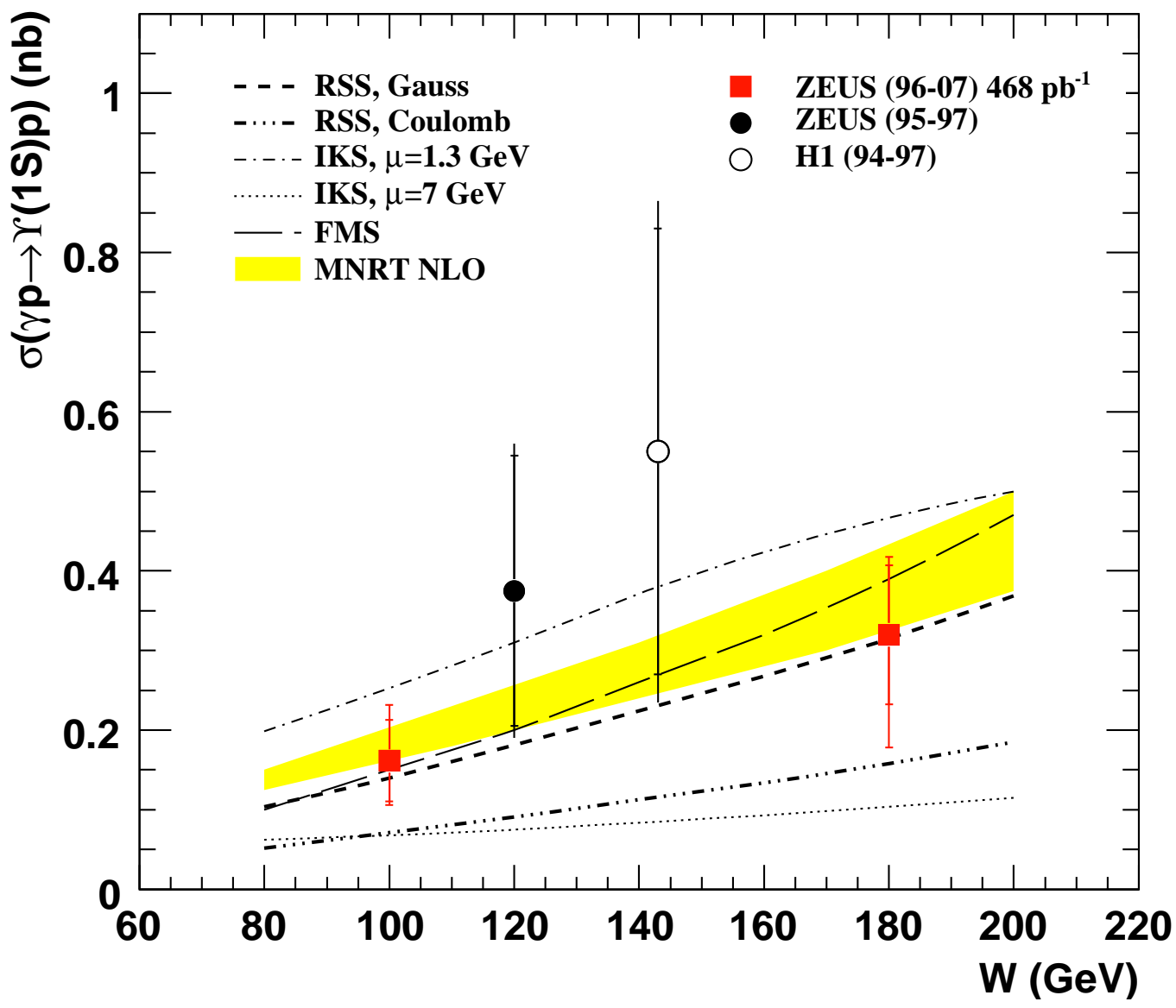

Figure 2: The exclusive $\Upsilon(1 \mathrm{~S})$ photoproduction cross section as a function of $W$. The full squares are the ZEUS data from this measurement in the kinematic region $Q^{2}<1 \mathrm{GeV}^{2}$, and two W ranges, $60<W<130 \mathrm{GeV}$, and $130<W<220 \mathrm{GeV}$. The inner bars indicate the statistical uncertainties, the outer bars are the statistical and systematic uncertainties added in quadrature. The earlier measurements of ZEUS [2] and H1 [3], are also shown. The shaded area denotes predictions of NLO MNRT [6]. The long-dashed line is the prediction of the FMS model [5]. The dashed-dotted (dotted) line is the prediction of the IKS [7] using a scale of 1.3 GeV ( $\left.{ }^{n} \mathrm{GeV}\right)$. The small-dashed line (small-dashed three-dots) is the prediction of $R S S$ [8], using a Gaussian-like (Coulomb-like) wave function. 